\title{
c-Abl-mediated tyrosine phosphorylation of DNA damage response proteins and implications in important cellular functions (Review)
}

\author{
AMEER ALI BOHIO ${ }^{1,2}$, RUOXI WANG ${ }^{1,2}$, XIANLU ZENG $^{1,2}$ and XUEQING BA ${ }^{1,2}$ \\ ${ }^{1}$ Key Laboratory of Molecular Epigenetics of The Ministry of Education, Institute of Genetics and Cytology; \\ ${ }^{2}$ School of Life Sciences, Northeast Normal University, Changchun, Jilin 130024, P.R. China
}

Received August 22, 2019; Accepted April 7, 2020

DOI: $10.3892 / \mathrm{mmr} .2020 .11156$

\begin{abstract}
Tyrosine phosphorylation is an essential post-translational protein modification catalyzed by tyrosine kinases. $\mathrm{c}-\mathrm{Abl}$ is a crucial non-receptor tyrosine kinase, which is most commonly activated by auto-phosphorylation, DNA damage and by interacting with other protein kinases. DNA damage response (DDR) proteins stimulated by DNA lesions or chromatin alterations recruit the DNA repair and cell cycle checkpoint machinery to restore genome integrity and cellular homeostasis. The fundamental roles of activated c-Abl tyrosine kinase in cellular response pathways have been intensively and extensively investigated and in recent years, a number of c-Abl protein binding partners have been determined; however, the functional roles of these molecules remain to be determined. The present review aimed to summarize the DDR proteins phosphorylated by c-Abl tyrosine kinase that have been identified to date, in addition to the functional outcomes of these phosphotyrosine events. Notably, it has been discovered that c-Abl tyrosine kinase can bind with and phosphorylate DDR proteins at different tyrosine sites, which serve distinct roles in various cellular contexts.
\end{abstract}

\section{Contents}

1. Introduction

2. Tyrosine phosphorylation

3. c-ABL tyrosine kinase

4. DNA damage response

Correspondence to: Professor Xueqing Ba, Key Laboratory of Molecular Epigenetics of The Ministry of Education, Institute of Genetics and Cytology, Northeast Normal University, 5268 Renmin Street, Changchun, Jilin 130024, P.R. China

E-mail: baxq755@nedu.edu.cn

Key words: c-Abl, tyrosine phosphorylation, DNA damage response proteins
5. Cellular functions regulated by the c-ABL-mediated tyrosine phosphorylation events of DNA damage response proteins

6. Conclusion

\section{Introduction}

Tyrosine phosphorylation is a post-translational protein modification, which serves as a rapid on/off switch for cellular machinery under regulated conditions. It is used in cell signaling pathways to regulate cell growth, migration, adhesion, differentiation and cell survival (1). This protein modification is regulated by tyrosine kinases; c-Abl tyrosine kinase is a crucial non-receptor tyrosine kinase responsible for the tyrosine phosphorylation of several proteins, mostly members of the DNA repair machinery (1). c-Abl has been associated with different aspects of the DNA damage response (DDR) and it is considered a major player in this area (1). c-Abl binds with various nuclear proteins implicated in different aspects of DNA repair, which suggests that c-Abl may be associated with the regulation of double-strand break repair (2). The role of c-Abl in DNA repair mechanisms first came into the spotlight in female germ cells under conditions of genotoxic stress (2). c-Abl was also discovered to be activated in cells upon DNA damage, whereby it successively activated signal transduction networks required to reinstate cellular homeostasis (3-7). It has since been found that DDR proteins are targets of $\mathrm{c}-\mathrm{Abl}$, similar to other non-receptor tyrosine kinases (8-11). c-Abl was also observed to translocate between the cytosol and nucleus, which revealed its important intermediary role in cellular signaling, capable of transducing signals from activated tyrosine kinases directly to the nuclear compartment to activate particular DDR pathways (8-11). The present review aimed to summarize the different roles of $\mathrm{c}-\mathrm{Abl}$ substrates belonging to the DDR machinery. These substrates are phosphorylated by c-Abl tyrosine kinase and are involved in different cellular functions, such as DNA damage repair, cell survival, tumor suppression, apoptosis, cell cycle arrest, DNA damage regulation and immune responses (Table I). In addition, the current review discussed the tyrosine phosphorylation events of DDR proteins mediated by c-Abl tyrosine kinase and summarized their implications in important cellular functions. 


\section{Tyrosine phosphorylation}

Historical overview. Post-translational protein modifications (for example, tyrosine phosphorylation) were first identified in 1979; however, it is now known that post-translational modifications have occurred for over a billion years throughout the evolution of unicellular eukaryotic organisms, the first multiple-celled eukaryotic animals (12). As regulated cell signal transduction is essential for multicellular organisms, the development of cell surface receptor systems that use tyrosine phosphorylation for transmembrane signal transduction and intracellular signaling seems likely to have been an important event in the evolution of multicellular organisms (12). Since the discovery of these post-translational modifications, rapid progress has been made in understanding the function of tyrosine phosphorylation. For example, the early observation that ligand binding induced a rapid increase in the autophosphorylation of epithelial growth factor and platelet-derived growth factor receptor tyrosine kinases suggested an important role for tyrosine phosphorylation in growth factor signaling and proliferation; by extension, this function was also suggested to be exploited in oncogenesis, through the hijacking of the growth factor tyrosine phosphorylation signaling pathways (13).

Significance of tyrosine phosphorylation and signaling mechanisms. Tyrosine phosphorylation is a post-translational protein modification, which is considered to be a major signaling transduction and regulatory mechanism in all eukaryotic cells. Tyrosine phosphorylation governs a number of cellular processes, such as cell differentiation, cell proliferation, transcriptional activation, neural transmission, cell cycle progression, aging, metabolic homeostasis and development (14). In fact, abnormal tyrosine phosphorylation, which is responsible for a number of human diseases, including cancer, has prompted the development of tyrosine kinase inhibitors (14). Tyrosine kinases serve a prominent role in cellular differentiation and development in metazoans; in evolutionary terms, tyrosine kinases are present in all metazoans, where they have been discovered to serve a major role in cell-cell signaling and transmembrane signal transduction, a crucial function for the establishment of a multicellular organism (14). The regulation of proteins by tyrosine phosphorylation occurs via several mechanisms, such as promoting electrostatic repulsion and inducing allosteric transitions; however, the most important role of tyrosine phosphorylation is to function as a docking site that supports specific interactions between a tyrosine phosphorylated protein and the protein containing a phosphotyrosine-binding (PTB) domain, such as the Src homology 2 (SH2) domain (14).

Docking interactions are crucial for the signal transduction downstream from receptor tyrosine kinases on the cell surface, which are activated upon the binding of a related extracellular ligand and in response, elicit specific cellular outcomes (12). Thousands of phosphotyrosine sites have been discovered; however, the function of tyrosine phosphorylation events occurring at these sites have not been investigated to date, to the best of our knowledge (12). Therefore, it is possible that a large number of these sites do not have a functional impact and may be referred as "noise" tyrosine phosphorylation events (12). In conclusion, this protein modification has been revealed to regulate a number of cellular functions and is often involved in oncogenesis (15).

\section{3. c-Abl tyrosine kinase}

Structural characteristics of $c$-Abl. c-Abl is a non-receptor tyrosine kinase that belongs to the ABL family of tyrosine kinases, which are located in both the cytoplasm and nucleus of the cell (16). c-Abl tyrosine kinase has been discovered to serve distinct roles in the cytoplasm and nucleus (17). The c-Abl protein possesses several defined domains, including the $\mathrm{SH} 2$ domain, which is located at the N-terminus of c-Abl and has a high level attraction for phosphorylated tyrosine residues, and the Src homology 3 (SH3) domain, which preferentially binds to proline-rich domains containing a PxxP motif. Interestingly, the identification of c-Abl SH3 binding proteins has proved useful for defining its close downstream proteins targets. c-Abl is also characterized by its long C-terminal tail, which contains both nuclear localization and nuclear export signals that regulate the c-Abl subcellular localization. The C-terminal tail also contains a DNA-binding domain, as well as F-actin and G-actin binding domains. Thus, through direct protein-protein interactions, c-Abl (with its structural domains) is likely to be simultaneously involved in a number of processes $(1,17,18)$.

Mechanisms of c-Abl activation. c-Abl is a tyrosine kinase and it is activated in response to several extracellular and intracellular stimuli (1). Generally, c-Abl exists in an inactive form in the cell, and the activity of c-Abl is strongly regulated by intramolecular bonds and by binding to other proteins, as well as by linkage to the membranes via an amino terminal myristoyl group. DNA damage, autophosphorylation and the actions of other proteins have all been reported to stimulate the activation of c-Abl $(16,19)$. The production of reactive oxidative species increases following DNA damage during cell stress, which may be responsible for cell degeneration (17). In addition, multiple cell processes are influenced by c-Abl through its interaction with other protein kinases and it has been suggested that the prime protein targets of $\mathrm{c}-\mathrm{Abl}$ may differ in various cell types (16). The activity of c-Abl has also been discovered to be strongly regulated and activated by ionizing radiation, as well as different types of genotoxic offence. According to previous studies, c-Abl has also been found to be activated by an unclear, slow and gradual mechanism $(1,20,21)$.

Downstream effects of $c$-Abl activation. The consensus sequence for the target phosphorylation of $\mathrm{c}-\mathrm{Abl}$ is non-promiscuous. Therefore, novel protein targets of $\mathrm{c}-\mathrm{Abl}$ should be experimentally verified under actual physiological conditions. Previously, c-Abl was investigated as a regulator of different types of human cancer and mutations in c-ABL were found to accelerate protein kinase activity, which was responsible for the high levels of cell proliferation (16). A number of c-Abl protein binding partners have been investigated; however, the functional outcomes of these compounds remain to be determined. To investigate c-Abl function, it is often beneficial to detect its immediate downstream partners. For instance, this method has led to the identification of a number 
Table I. c-ABL substrates belonging to the DNA damage response machinery: Phosphorylation sites and functional roles.

\begin{tabular}{|c|c|c|c|c|}
\hline Authors (year) & c-ABL substrate & $\begin{array}{l}\text { Phosphotyrosine } \\
\text { site }\end{array}$ & Function & (Refs.) \\
\hline Chen et al (1999) & & & & $(41)$ \\
\hline Shimizu et al (2009) & RAD51 & 315 & DNA recombination, repair and survival & (42) \\
\hline Kitao and Yuan (2002) & RAD52 & 104 & DNA recombination, repair and survival & (43) \\
\hline Goldberg et al (2002) & & & & (46) \\
\hline Carr et al (2016) & Mouse double minute 2 & 394 & Tumor suppression and apoptosis & (47) \\
\hline Dias et al (2006) & $\begin{array}{l}\text { HDM2 (human homolog } \\
\text { of MDM2) }\end{array}$ & 276 & Tumor suppression and apoptosis & (48) \\
\hline Chen et al (2016) & & & & (49) \\
\hline Xiong et al (2006) & MDMX (MDM4) & 99 & Tumor suppression and apoptosis & (50) \\
\hline Reuven et al (2015) & $\begin{array}{l}\text { Homeodomain-interacting } \\
\text { protein kinase } 2\end{array}$ & 360 & Tumor suppression and apoptosis & (4) \\
\hline Gonfloni et al (2009) & p63 (ТР63) & 149 & Tumor suppression and apoptosis & (53) \\
\hline Yuan et al (1999) & P73 & 99 & Tumor suppression and apoptosis & (54) \\
\hline Keshet et al (2015) & Yes-associated protein 1 & 357 & Tumor suppression and apoptosis & (55) \\
\hline Yamaguchi et al (2015) & & & & (56) \\
\hline Karimian et al (2016) & Jun B & $\begin{array}{c}173,182 \\
\& 188\end{array}$ & Cell cycle arrest & (57) \\
\hline Jin et al (1997) & & & & (58) \\
\hline Hartley et al (1995) & & & & (59) \\
\hline $\begin{array}{l}\text { Keith and Schreiber } \\
\text { (1995) }\end{array}$ & $\begin{array}{l}\text { DNA-dependent protein } \\
\text { kinase, catalytic subunit }\end{array}$ & $\begin{array}{l}\text { pYC-terminal } \\
\text { domain }\end{array}$ & DNA damage regulation & (60) \\
\hline Yi et al (2006) & $\begin{array}{l}\text { Mut S protein } \\
\text { homology } 5\end{array}$ & $\begin{array}{l}\mathrm{NH} 2 \text { terminus } \\
\text { ' } 1-109\end{array}$ & DNA damage regulation & (61) \\
\hline Foray et al (2002) & $\begin{array}{l}\text { Breast cancer type } 1 \\
\text { susceptibility protein }\end{array}$ & $\begin{array}{c}\text { C-terminal } \\
\text { region } 1,314-1 \text {, } \\
863\end{array}$ & c-ABL kinase activation & (63) \\
\hline Bohio et al (2019) & $\begin{array}{l}\text { Poly (ADP-ribose) } \\
\text { polymerase } 1\end{array}$ & 829 & Immune response & (69) \\
\hline
\end{tabular}

of proteins, including TP53 and RAD51, which are members of the DNA repair machinery (17); c-Abl is capable of binding to several of these proteins (22). c-Abl has been found to be part of an intricate network of protein interactions, as well as phosphorylation events, in the cell and it is expressed in all types of cells. Therefore, it was hypothesized that c-Abl is involved in a variety of physiological functions, such as cell growth, cell survival, autophagy, DNA repair, motility, cytoskeleton dynamics and receptor endocytosis (16). c-Abl has also been discovered to enhance the activity of a number of substrates to regulate DNA double strand break repair pathways $(17,23)$. In addition, c-Abl has been implicated in various cell signaling pathways, including those originating from cell adhesion, DNA damage, growth factor stimulation and oxidative stress (24-27). c-Abl has been found to support the DNA damage repair mechanism; however, it has been discovered to promote apoptosis in circumstances when DNA damage repair is not possible (28).

\section{DNA damage response}

DNA damage and repair mechanism. DNA damage occurs following the exposure to environmental (UV light and ionizing radiation) or intracellular (reactive oxygen species as byproducts of routine metabolic processes) genotoxic agents (29). Fortunately, cells are equipped with several DNA repair mechanisms, including base excision repair (BER) that removes damaged bases, mismatch repair that recognizes base incorporation errors and base damage, nucleotide excision repair that removes bulky DNA adducts and cross-link repair that removes interstrand cross-links. Breaks in the DNA backbone are repaired by double-strand break repair signaling pathways, including homologous recombination and non-homologous end joining (30). Several of these mechanisms can operate independently to repair simple lesions, for instance, BER removes base lesions including, and oxidation (31). DNA glycosylases scan the DNA in 


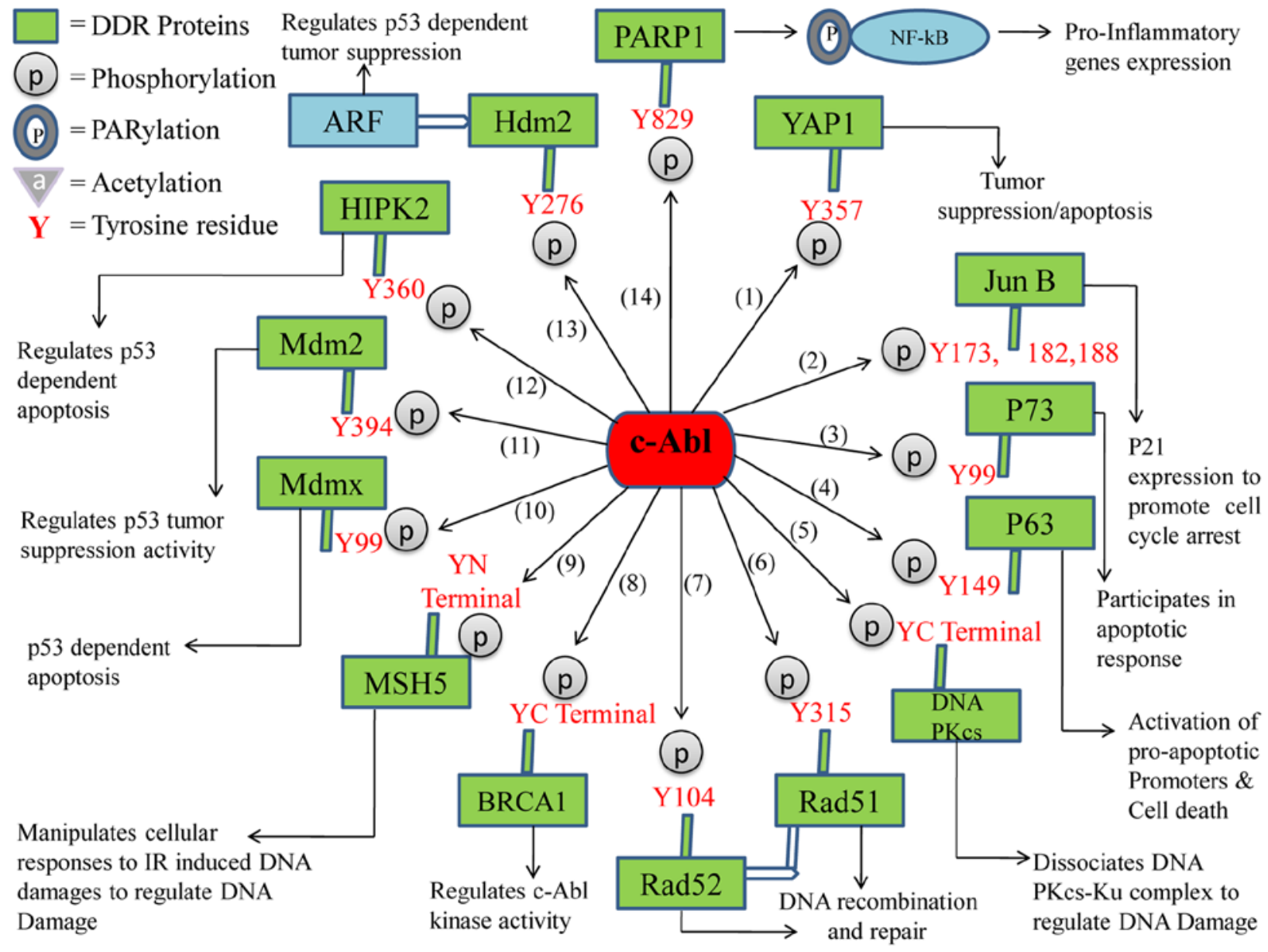

Figure 1. Schematic diagram of the c-ABL-mediated phosphotyrosine events of DDR proteins and the functional outcomes. (1) The phosphorylation of YAP1 on Y357 inactivates the oncogenic function of YAP1, and regulates tumor suppression and apoptosis. (2) Phosphorylated Jun B at Y173, 182,188 initiates the expression of p21 to promote cell cycle arrest. (3) P73 phosphorylation on Y99 increases apoptosis. (4) p63 phosphorylation on Y149 activates proapoptotic promoters and regulates cell death. (5) Tyrosine phosphorylation of DNA-PKcs at the C-terminal region results in the dissociation of the DNA-PKcs-Ku complex and the regulation of DNA damage. (6) Phosphorylated RAD51 on Y315 promotes the formation of the complex between RAD51 and RAD52 protein, which cooperates with RAD51 in DNA recombination and repair. (7) Phosphorylated RAD52 on Y104 regulates DNA recombination and repair. (8) BRCA1 tyrosine phosphorylation at the C-terminal region regulates c-ABL kinase activity. (9) Phosphorylated MSH5 at the N-terminal region manipulates cellular responses to IR-induced DNA damages to regulate DNA damage. (10) MDMX phosphorylation at Y99 regulates p53 dependent apoptosis. (11) MDM2 phosphorylation at Y394 regulates p53 tumor suppressor activity. (12) HIPK2 phosphorylation at Y360 regulates p53 dependent apoptosis. (13) Phosphorylation of HDM2 at Y276 regulates the interaction with ARF that contributes functionally to the induction of p53 and tumor suppression. (14) Phosphorylated PARP1 at the Y829 site results in PARP1/NF- $\kappa$ B signaling pathway dependent proinflammatory gene expression and inflammation. DDR, DNA damage response; YAP1, yes-associated protein 1; DNA-PKcs, DNA-dependent protein kinase, catalytic subunit; BRCA1, breast cancer type 1 susceptibility protein; MSH5, mut S protein homology 5; MDM2, mouse double minute 2; HIPK2, homeodomain-interacting protein kinase 2; HDM2, human homolog of MDM2; alternate reading frame protein product of the INK4a/Arf locus, p14 ${ }^{\mathrm{ARF}}$; PARP1, poly (ADP-ribose) polymerase 1; IR, ionizing radiation.

order to recognize and remove the damage, along with other proteins, such as AP endonuclease 1, DNA polymerase $\beta$ and DNA ligase in a 'hand-off' model, in order to restore the DNA duplex (31). However, the DDRs, such as DSB response, regulate the repair of more complex lesions, which involves multiple DNA processing steps. DDR is considered to be essential for the successful repair of the more difficult lesions (30). In stable or normal cells, genomic integrity is maintained by a strong and effective DDR signaling network, which includes DNA repair signaling pathways and cell cycle checkpoints (29). The repair of DNA damage is necessary for RNA and DNA polymerases to accurately read and transcribe the information in the genome. During double-strand breaks, multiple enzymes and mediators translocate to the sites of the lesion to release a network of DNA repair processes known as the DDR (2). The functions of the DDR and TP53 were found to be necessary for the determination of cell fate in gastric cancer (32). In addition, tumor necrosis factor receptor associated factor 1 interacting protein was discovered to be a novel regulator of histone $\mathrm{H} 2 \mathrm{~B}$ monoubiquitination in the DDR and during cancer development in lung adenocarcinoma (33).

Role of protein kinases in the DDR. DNA damage arising from both internal and environmental sources challenges the integrity of the genome. DDR protein kinases have been discovered to regulate these repair mechanisms (30). These kinases phosphorylate DNA repair proteins, and they have been found to increase the efficiency of DNA repair by initiating a series of multiple changes in the local chromatin structure near the damage site and by changing the overall environment of the cell to make it more efficient for repair (30). The DNA damage response kinases were indicated to share multiple common 
regulatory mechanisms of activation (34), for example, they sense DNA damage through protein-protein interactions that serve to recruit kinases to the sites of damage. Once recruited, protein-protein interactions and post-translational modifications fully activate the kinases to begin a cascade of phosphorylation events $(35,36)$.

Role of $c$-Abl in the DDR. The c-Abl tyrosine kinase is activated by DNA damaging agents (ionizing radiation), alkylating agents (mitomycin C) and DNA cross-linking agents (cisplatin); these triggering factors initiate a strong c-Abl response to DNA damage signaling (1). The ataxia-telangiectasia-mutated (ATM) protein is an upstream effector of c-Abl and binds with the c-Abl SH3 domain (1). c-Abl is phosphorylated by ATM at the serine site of 465 following ionizing radiation in cells, which results in c-Abl activation. Therefore, in response to DNA damage, ATM serves a crucial role in c-Abl activation $(1,18)$. DNA transaction processes (DNA replication, recombination and repair) serve a basic role in the stability of the genome and diversity. It has been discovered that c-Abl is involved in these processes through its DNA binding domain (17). A number of auxiliary proteins, enzymes and effectors participating in DNA transactions have been observed to bind with, and be modified, by the c-Abl tyrosine kinase (17). In fact, c-Abl has been discovered to bind with and phosphorylate several DDR proteins $(1,37,38)$; therefore, it is suggested that the DDR is regulated by $\mathrm{c}-\mathrm{Abl}$. In addition, the tyrosine kinase $\mathrm{c}-\mathrm{Abl}$ may act as a decision maker in the cellular environment due to its role in the activation of the proapoptotic signaling pathway in circumstances when DNA damage repair is not possible $(1,37,38)$.

\section{Cellular functions regulated by the c-Abl-mediated tyrosine phosphorylation events of DNA damage response proteins}

c-Abl tyrosine kinase is an unique kinase activated by genotoxic stress in cells, which directly phosphorylates various major cellular proteins, including DDR proteins to regulate important cellular functions in the cell (Fig. 1) $(39,40)$. Here in, these DDR proteins are categorized according to their functional roles.

c-Abl targets proteins involved in DNA recombination, repair and survival

$R A D 51 . \mathrm{RAD} 51$ is a DNA recombination and repair protein, which is a homologue of the bacterial RecA protein. The c-Abl-dependent tyrosine phosphorylation of RAD51 is dependent on ATM, which is activated following ionizing radiation. c-Abl, ATM and RAD51 can be co-immunoprecipitated from cell extracts, suggesting the ATM/c-Abl signaling pathway is responsible for the correct post-translational modification of RAD51, which is necessary for the assembly of the RAD51 repair protein complex following ionizing radiation. Specifically, DNA damage-activated c-Abl tyrosine kinase phosphorylates RAD51 on tyrosine site 315 and this phosphorylation induces the formation of the RAD51/RAD52 protein complex, which assists RAD51 in DNA recombination and repair $(41,42)$.
$R A D 52$. The RAD52 protein plays an important role in the homologous recombination repair of double-strand breaks in collaboration with RAD51 and RAD54, which are all known as the epistasis group of proteins. A well-known feature linked with the ability of these proteins to repair double-strand breaks is the formation of inducible nuclear foci at damaged sites. c-Abl tyrosine kinase has been discovered to bind with, and phosphorylate RAD52 on tyrosine site 104 in cells following ionizing radiation (43).

\section{c-Abl targets proteins involved in tumor suppression and apoptosis}

Mouse double minute 2 (MDM2). MDM2 has been established to be a major negative regulator of the tumor suppressor protein p53, which promotes tumor formation $(43,44)$. In response to DNA damage, c-Abl interacts with and phosphorylates MDM2 at the tyrosine site Y394; the c-Abl-dependent tyrosine phosphorylation of MDM2 neutralizes the MDM2-induced inhibitory effects over p53. In fact, the phosphorylation of MDM2 by c-Abl has been found to regulate the p53 tumor suppressive activity both in vitro and in vivo $(45,46)$.

Human homologue of MDM2 (HDM2). HDM2 is a major negative regulator of the tumor suppressor protein, $\mathrm{p} 53$, which can promote tumor formation (47). In response to DNA damage, c-Abl interacts with HDM2 and induces its phosphorylation at tyrosine site Y276. The c-Abl-dependent tyrosine phosphorylation of HDM2 subsequently regulates its interaction with an alternate reading frame protein product of the INK4a/Arf locus, known as p14 ${ }^{\mathrm{ARF}}$, which results in an increased level of nucleolar HDM2 and a low turnover of p53. Thus, indicating that the c-Abl-induced phosphorylation of HDM2 tyrosine site Y276 is involved in the induction of p53 and regulation of p53-dependent tumor suppression (48).

MDMX (MDM4). MDMX is a homologue of the E3 ubiquitin ligase MDM2 protein. MDMX has been discovered to negatively regulate the tumor suppressor protein p53 in normal cells, through both MDM2-dependent and -independent mechanisms. c-Abl tyrosine kinase phosphorylates MDMX at tyrosine site 99 following DNA damage, which subsequently disrupts the binding of MDMX to p53 and as a result, p53 escapes MDMX- and MDM2-dependent functional inhibition and degradation. Therefore, the phosphorylation of MDMX by c-Abl protects damaged cells from proliferation through the induction of cell cycle arrest, DNA repair, senescence or programmed cell death $(49,50)$.

Homeodomain-interacting protein kinase 2 (HIPK2). HIPK2 is an important regulator of p53-dependent programmed cell death. Several sites of HIPK2 have been discovered to be phosphorylated by c-Abl, which protects HIPK2 from ubiquitin E3 ligase Siah-1-mediated degradation. The tyrosine phosphorylation of HIPK2 at tyrosine residue 360 (Y360) by c-Abl occurs following DNA damage, which regulates p53-dependent programmed cell death (4).

Tumor protein 63 (TP63)/p63. p63 or TP63 belongs to the p53 family of tumor-suppressor proteins $(51,52)$. c-Abl has been discovered to phosphorylate p63 on the Y149 tyrosine residue 
following cisplatin treatment in vitro. The subsequent modification has been revealed to affect p63 stability and induce the p63-dependent activation of proapoptotic promoters. In fact, the activation of the c-Abl/p63 signaling pathway by chemotherapeutic DNA-damaging drugs has been observed in model human cell lines and mouse oocytes, where it plays an important role in apoptosis (53).

p73. The p73 protein belongs to the p53 family of tumor-suppressor proteins and it can also induce programmed cell death. c-Abl was revealed to interact with the carboxy-terminal homo-oligomerization domain of the p73 protein through the SH3 domain. Following ionizing radiation, p73 is phosphorylated by the c-Abl tyrosine kinase on the tyrosine site of 99 both in vitro and in vivo. Importantly, the p73-mediated transactivation and apoptosis have both been found to be regulated by c-Abl. In response to DNA damage, the c-Abl-mediated regulation of p73 has also been observed with the failure of ionizing radiation-induced apoptosis following the interruption of the $\mathrm{c}-\mathrm{Abl} / \mathrm{p} 73$ interaction. Thus, a c-Abl-dependent mechanism regulating both p53 and p73 is suggested to be involved in the apoptotic response to DNA damage (54).

YES-associated protein 1 (YAP1). YAP1 has been discovered to function as an oncogene in a number of experimental systems, as well as functioning as a central transcription co-activator. The transcriptional enhancer activator domain (TEAD) family of transcription factors are known to be co-activated by YAP. The tyrosine kinase c-Abl and DNA damage has been discovered to serve as negative regulators of TEAD co-activation through YAP; c-Abl tyrosine kinase phosphorylates YAP1 at the Y357 residue under conditions of DNA damage. This phosphorylation event results in the activation of proapoptotic genes in relation to $\mathrm{p} 73$ and inactivates the oncogenic function of YAP1 (55).

\section{c-Abl targets proteins involved in cell cycle arrest}

Jun B. Jun B belongs to the activator protein 1 (AP1) transcription factor family and it has since been discovered to be associated with the DDR. c-Abl was found to phosphorylate Jun B at the tyrosine sites of 173, 182 and 188, which inhibits its suppressive role in $\mathrm{p} 21$ promoter activity, most likely through blocking the formation of the Jun B/c-Fos AP1 complex. c-Abl targets Jun B, which is required for Adriamycin-induced expression of p21 (56). In response to a variety of stimuli, p21 has been found to promote cell cycle arrest (57).

\section{c-Abl targets proteins involved in DNA damage regulation} DNA-dependent protein kinase, catalytic subunit (DNA-PKcs). DNA-PKcs is the $470 \mathrm{kDa}$ catalytic subunit of DNA-dependent protein kinase (DNA-PK). In mammalian cells, the double-stranded DNA break repair mechanism is controlled by DNA-PKcs. The structure of DNA-PKcs is similar to multiple other proteins involved in radiation-induced checkpoint responses. DNA-PKcs target DNA breaks together with the DNA binding heterodimer, $\mathrm{Ku}$ (58). It was reported that the C-terminal fragments of DNA-PKcs were phosphorylated by c-Abl, specifically the amino acids 3,414 to 3,850 . The c-Abl-mediated phosphorylation of DNA-PKcs was discov- ered to subsequently disrupt the DNA-PKcs and Ku complex. Therefore, $\mathrm{c}-\mathrm{Abl}$ and $\mathrm{Ku}$ may perform opposing functions with relation to regulating DNA-PK activity (58-60).

MutS protein homolog 5 (MSH5). MSH5 belongs to the DNA mismatch repair family of proteins. Human MSH5 and c-Abl are associated by the direct physical interaction between the NH2 terminus (residues 1-109) of human MSH5 (hMSH5) and the c-Abl SH3 domain. The activation of c-Abl tyrosine kinase and the phosphorylation of hMSH5 in response to ionizing radiation were found to be dependent upon this physical interaction. This interaction of hMSH5 with c-Abl also implied that the crosstalk between hMSH5 and c-Abl may manipulate the cellular responses to ionizing radiation-induced DNA damage (61).

\section{c-Abl kinase activation}

Breast cancer type 1 susceptibility protein (BRCA1). BRCA1 is expressed in breast cells, as well as in other tissues (62). BRCA1 supports DNA repair; however, it also destroys cells in circumstances when DNA repair is not possible (62). c-Abl was discovered to bind to the C-terminal region of BRCA1 (residues 1,314 to 1,863) and phosphorylate the C-terminus in vitro. In addition, BRCA1 was also found to be phosphorylated at tyrosine residues in an ATM- and radiation-dependent manner. The tyrosine phosphorylation of BRCA1 is not required to disrupt the complex between BRCA1/c-Abl; the complex co-binds essentially; however, the exposure to ionizing radiation triggers an ATM-dependent disruption of the BRCA1/c-Abl complex, which coincides with the activation of c-Abl kinase activity. In fact, in one previous study, c-Abl kinase activity was found to be constitutively elevated following the loss of BRCA1 (63).

\section{c-Abl targets proteins involved in the immune response} Poly (ADP-ribose) polymerase 1 (PARP1). PARP1 is a crucial DDR protein $(64,65)$. PARP1 has been observed to be activated by DNA damage, including strand breaks and apurinic/apyrimidinic sites, and is thus physiologically associated with DNA damage detection, DNA repair and cell death (66-68). According to a recent study by our group, upon immunological challenges, $\mathrm{c}$-Abl was found to undergo nuclear translocation, and bind with and phosphorylate PARP1 at the conserved tyrosine site, Y829. The c-ABL-mediated tyrosine phosphorylation of PARP1 resulted in the activation of a PARP1/NF- $\kappa \mathrm{B}$ signaling pathway-dependent immune response by inducing proinflammatory gene expression (69).

\section{Conclusion}

In conclusion, $\mathrm{c}-\mathrm{Abl}$ has been discovered to interact with DDR proteins to dictate cell fate/DNA repair, transcriptional regulation, tumor suppression, cell death or cell cycle arrest following DNA damage. The present review presented an overview of all DDR proteins phosphorylated by c-Abl tyrosine kinase and the functional outcomes of these phosphotyrosine events. c-Abl tyrosine kinase was found to phosphorylate DDR proteins on different tyrosine sites and these phosphotyrosine events of DDR proteins were reported to be crucial for the activation of other cell signaling pathways to regulate important cellular 
functions. Altogether, this review provided evidence to suggest that the pharmacological inhibition of c-Abl may be of clinical use in certain physiopathological contexts.

\section{Acknowledgements}

The authors would like to thank Dr. Istvan Boldogh (Department of Microbiology and Immunology, University of Texas Medical Branch at Galveston) for his intellectual input in this review.

\section{Funding}

The present work was supported by grants from the National Natural Science Foundation of China (grant nos. 31970686 and 31571339 to XB), the Program for Introducing Talent to Universities (grant no. B07017 to XB) and the Natural Science Foundation of Jilin, China (grant no. 20180101236JC to XB).

\section{Availability of data and materials}

Not applicable.

\section{Authors' contributions}

$\mathrm{XB}$ and $\mathrm{XZ}$ decided on the topics discussed in the review; RW and $\mathrm{AAB}$ obtained the relevant literature; and $\mathrm{AAB}$ wrote the article, which was reviewed by XB. All authors read and approved the final manuscript and agree to be accountable for the accuracy and referencing of the information included in the review.

\section{Ethics approval and consent to participate}

Not applicable.

\section{Patient consent for publication}

Not applicable.

\section{Competing interests}

The authors declare that they have no competing interests.

\section{References}

1. Shaul Y and Ben-Yehoyada M: Role of c-Abl in the DNA damage stress response. Cell Res 15: 33-35, 2005.

2. Maiani E, Diederich M and Gonfloni S: DNA damage response: The emerging role of $\mathrm{c}-\mathrm{Abl}$ as a regulatory switch? Biochem Pharmacol 82: 1269-1276, 2011.

3. Tu CC, Zhong Y, Nguyen L, Tsai A, Sridevi P, Tarn WY and Wang JY: The kinase ABL phosphorylates the microprocessor subunit DGCR8 to stimulate primary microRNA processing in response to DNA damage. Sci Signal 8: ra64, 2015.

4. Reuven N, Adler J, Porat Z, Polonio-Vallon T, Hofmann TG and Shaul Y: The tyrosine kinase c-Abl promotes homeodomain-interacting protein kinase 2 (HIPK2) accumulation and activation in response to DNA damage. J Biol Chem 290: 16478-16488, 2015.

5. Staquicini FI, Qian MD, Salameh A, Dobroff AS, Edwards JK, Cimino DF, Moeller BJ, Kelly P, Nunez MI, Tang X, et al: Receptor tyrosine kinase EphA5 is a functional molecular target in human lung cancer. J Biol Chem 290: 7345-7359, 2015.
6. Chen E, Ahn JS, Massie CE, Clynes D, Godfrey AL, Li J, Park HJ, Nangalia J, Silber Y, Mullally A, et al: JAK2V617F promotes replication fork stalling with disease-restricted impairment of the intra-S checkpoint response. Proc Natl Acad Sci USA 111: 15190-15195, 2014.

7. Reuven N, Adler J, Meltser V and Shaul Y: The Hippo pathway kinase Lats2 prevents DNA damage-induced apoptosis through inhibition of the tyrosine kinase c-Abl. Cell Death Differ 20: 1330-1340, 2013.

8. Fukumoto Y, Morii M, Miura T, Kubota S, Ishibashi K, Honda T, Okamoto A, Yamaguchi N, Iwama A, Nakayama Y and Yamaguchi N: Src family kinases promote silencing of ATR-Chk1signaling in termination of DNA damage checkpoint. J Bol Chem 289: 12313-12329, 2014.

9. Mahajan K, Coppola D, Rawal B, Chen YA, Lawrence HR, Engelman RW, Lawrence NJ and Mahajan NP: Ack1-mediated androgen receptor phosphorylation modulates radiation resistance in castration-resistant prostate cancer. J Biol Chem 287: 22112-22122, 2012.

10. Kharbanda S, Saleem A, Yuan ZM, Kraeft S, Weichselbaum R, Chen LB and Kufe D: Nuclear signaling induced by ionizing radiation involves colocalization of the activated p56/p53lyn tyrosine kinase with p34cdc2. Cancer Res 56: 3617-3621, 1996.

11. Srinivasan D and Plattner R: Activation of Abl tyrosine kinases promotes invasion of aggressive breast cancer cells. Cancer Res 66: 5648-5655, 2006.

12. Hunter T: The genesis of tyrosine phosphorylation. Cold Spring Harb Perspect Biol 6: a020644, 2014.

13. Pawson T: Specificity in signal transduction: From phosphotyrosine-SH2 domain interactions to complex cellular systems. Cell 116: 191-203, 2004.

14. Hunter T: Tyrosine phosphorylation: Thirty years and counting. Curr Opin Cell Biol 21: 140-146, 2009.

15. Lind SB, Artemenko KA and Pettersson U: A strategy for identification of protein tyrosine phosphorylation. Methods 56: 275-283, 2012.

16. Hantschel O and Superti-Furga G: Regulation of the c-Abl and Bcr-Abl tyrosine kinases. Nat Rev Mol Cell Biol 5: 33-44, 2004.

17. Shaul Y: c-Abl: Activation and nuclear targets. Cell Death Differ 7: 10-16, 2000.

18. Shiloh Y: ATM and related protein kinases: Safeguarding genome integrity. Nat Rev Cancer 3: 155-168, 2003.

19. Lindholm D, Pham DD, Cascone A, Eriksson O, Wennerberg K and Saarma M: c-Abl inhibitors enable insights into the pathophysiology and neuroprotection in parkinson's disease. Front Aging Neurosci 8: 254, 2016.

20. Liu ZG, Baskaran R, Lea-Chou ET, Wood LD, Chen Y, Karin M and Wang JY: Three distinct signalling responses by murine fibroblasts to genotoxic stress. Nature 384: 273-276, 1996.

21. Kharbanda S, Ren R, Pandey P, Shafman TD, Feller SM, Weichselbaum RR and Kufe DW: Activation of the c-Abl tyrosine kinase in the stress response to DNA-damaging agents. Nature 376: 785-788, 1995.

22. Colicelli J: ABL tyrosine kinases: Evolution of function, regulation, and specificity. Sci Signal 3: re6, 2010.

23. Zuckerman V, Lenos K, Popowicz GM, Silberman I, Grossman T, Marine JC, Holak TA, Jochemsen AG and Haupt Y: c-Abl phosphorylates Hdmx and regulates its interaction with p53. J Biol Chem 284: 4031-4039, 2009.

24. Gu JJ, Ryu JR and Pendergast AM: Abl tyrosine kinases in T-cell signaling. Immunol Rev 228: 170-183, 2009.

25. Sirvent A, Benistant C and Roche S: Cytoplasmic signalling by the c-Abl tyrosine kinase in normal and cancer cells. Biol Cell 100: 617-631, 2008.

26. Zhu J and Wang JY: Death by Abl: A matter of location. Curr Top Dev Biol 59: 165-192, 2004.

27. Pendergast AM: The Abl family kinases: Mechanisms of regulation and signaling. Adv Cancer Res 85: 51-100, 2002.

28. Gonfloni S: DNA damage stress response in germ cells: Role of c-Abl and clinical implications. Oncogene 29: 6193-6202, 2010.

29. Curtin NJ: DNA repair dysregulation from cancer driver to therapeutic target. Nat Rev Cancer 12: 801-817, 2012.

30. Sirbu BM and Cortez D: DNA damage response: Three levels of DNA repair regulation. Cold Spring Harb Perspect Biol 5: a012724, 2013

31. Ba X and Boldogh I: 8-Oxoguanine DNA glycosylase 1: Beyond repair of the oxidatively modified base lesions. Redox Biol 14: 669-678, 2018. 
32. Arai $H$, Wada $R$, Ishino $K$, Kudo $M$, Uchida $E$ and Naito $Z$ : Expression of DNA damage response proteins in gastric cancer: Comprehensive protein profiling and histological analysis. Int J Oncol 52: 978-988, 2018.

33. Han YG, Yun M, Choi M, Lee SG and Kim H: TRAIP regulates Histone H2B monoubiquitination in DNA damage response pathways. Oncol Rep 41: 3305-3312, 2019.

34. Lovejoy CA and Cortez D: Common mechanisms of PIKK regulation. DNA Repair (Amst) 8: 1004-1008, 2009.

35. Weterings E and Chen DJ: DNA-dependent protein kinase in nonhomologous end joining: A lock with multiple keys? J Cell Biol 179: 183-186, 2007.

36. Dobbs TA, Tainer JA and Lees-Miller SP: A structural model for regulation of NHEJ by DNA-PKcs autophosphorylation. DNA Repair (Amst) 9: 1307-1314, 2010

37. Kharbanda S, Yuan ZM, Weichselbaum R and Kufe D: Functional role for the c-Abl protein tyrosine kinase in the cellular response to genotoxic stress. Biochim Biophys Acta 1333: O1-O7, 1997.

38. Wang JY: Controlling Abl: Auto-inhibition and co-inhibition? Nat Cell Biol 6: 3-7, 2004.

39. Tang J, Wang JY and Parker LL: Detection of early Abl kinase activation after ionizing radiation by using a peptide biosensor. Chembiochem 13: 665-673, 2012.

40. Meltser V, Ben-Yehoyada M and Shaul Y: c-Abl tyrosine kinase in the DNA damage response: Cell death and more. Cell Death Differ 18: 2-4, 2011.

41. Chen G, Yuan SS, Liu W, Xu Y, Trujillo K, Song B, Cong F, Goff SP, Wu Y, Arlinghaus R, et al: Radiation-induced assembly of Rad51 and Rad52 recombination complex requires ATM and c-Abl. J Biol Chem 274: 12748-12752, 1999.

42. Shimizu H, Popova M, Fleury F, Kobayashi M, Hayashi N, Sakane I, Kurumizaka H, Venkitaraman AR, Takahashi M and Yamamoto K: c-ABL tyrosine kinase stabilizes RAD51 chromatin association. Biochem Biophys Res Commun 382: 286-291, 2009.

43. Kitao $\mathrm{H}$ and Yuan ZM: Regulation of ionizing radiation-induced Rad52 nuclear foci formation by c-Abl-mediated phosphorylation. J Biol Chem 277: 48944-48948, 2002.

44. Oliner JD, Kinzler KW, Meltzer PS, George DL and Vogelstein B Amplification of a gene encoding a p53-associated protein in human sarcomas. Nature 358: 80-83, 1992.

45. Goldberg Z, Vogt Sionov R, Berger M, Zwang Y, Perets R, Van Etten RA, Oren M, Taya Y and Haupt Y: Tyrosine phosphorylation of Mdm2 by c-Abl: Implications for p53 regulation. EMBO J 21: 3715-3727, 2002.

46. Carr MI, Roderick JE, Zhang H, Woda BA, Kelliher MA and Jones SN: Phosphorylation of the Mdm2 oncoprotein by the c-Abl tyrosine kinase regulates p53 tumor suppression and the radiosensitivity of mice. Proc Natl Acad Sci USA 113 15024-15029, 2016.

47. Wade M, Wong ET, Tang M, Stommel JM and Wahl GM: Hdmx modulates the outcome of 553 activation in human tumor cells. J Biol Chem 281: 33036-33044, 2006.

48. Dias SS, Milne DM and Meek DW: c-Abl phosphorylates Hdm2 at tyrosine 276 in response to DNA damage and regulates interaction with ARF. Oncogene 25: 6666-6671, 2006.

49. Chen X, Gohain N, Zhan C, Lu WY, Pazgier M and Lu W: Structural basis of how stress-induced MDMX phosphorylation activates p53. Oncogene 35: 1919-1925, 2016.

50. Xiong S, Van Pelt CS, Elizondo-Fraire AC, Liu G and Lozano G: Synergistic roles of Mdm2 and Mdm4 for p53 inhibition in central nervous system development. Proc Natl Acad Sci USA 103: 3226-3231, 2006.

51. Yang A, Kaghad M, Wang Y, Gillett E, Fleming MD, Dötsch V, Andrews NC, Caput D and McKeon F: p63, a p53 homolog at 3q27-29, encodes multiple products with transactivating, death-inducing, and dominant-negative activities. Mol Cell 2 : 305-316, 1998
52. Wu G, Nomoto S, Hoque MO, Dracheva T, Osada M, Lee CC, Dong SM, Guo Z, Benoit N, Cohen Y, et al: DeltaNp63alpha and TAp63alpha regulate transcription of genes with distinct biological functions in cancer and development. Cancer Res 63: 2351-2357, 2003

53. Gonfloni S, Di Tella L, Caldarola S, Cannata SM, Klinger FG, Di Bartolomeo C, Mattei M, Candi E, De Felici M, Melino G and Cesareni G: Inhibition of the c-Abl-TAp63 pathway protects mouse oocytes from chemotherapy-induced death. Nat Med 15: 1179-1185, 2009

54. Yuan ZM, Shioya H, Ishiko T, Sun X, Gu J, Huang YY, Lu H, Kharbanda S, Weichselbaum R and Kufe D: p73 is regulated by tyrosine kinase c-Abl in the apoptotic response to DNA damage. Nature 399: 814-817, 1999.

55. Keshet R, Adler J, Ricardo Lax I, Shanzer M, Porat Z, Reuven N and Shaul Y: c-Abl antagonizes the YAP oncogenic function. Cell Death Differ 22: 935-945, 2015.

56. Yamaguchi N, Yuki R, Kubota S, Aoyama K, Kuga T, Hashimoto Y, Tomonaga T and Yamaguchi N: c-Abl-mediated tyrosine phosphorylation of JunB is required for adriamycin-induced expression of p21. Biochem J 471: 67-77, 2015.

57. Karimian A, Ahmadi Y and Yousefi B: Multiple functions of p21 in cell cycle, apoptosis and transcriptional regulation after DNA damage. DNA Repair (Amst) 42: 63-71, 2016.

58. Jin S, Kharbanda S, Mayer B, Kufe D and Weaver DT: Binding of $\mathrm{Ku}$ and $\mathrm{c}-\mathrm{Abl}$ at the kinase homology region of DNA-dependent protein kinase catalytic subunit. J Biol Chem 272: 24763-24766, 1997.

59. Hartley KO, Gell D, Smith GC, Zhang H, Divecha N, Connelly MA, Admon A, Lees-Miller SP, Anderson CW and Jackson SP: DNA-dependent protein kinase catalytic subunit: A relative of phosphatidylinositol 3-kinase and the ataxia telangiectasia gene product. Cell 82: 849-856, 1995.

60. Keith CT and Schreiber SL: PIK-related kinases: DNA repair, recombination, and cell cycle checkpoints. Science 270: 50-51, 1995.

61. Yi W, Lee TH, Tompkins JD, Zhu F, Wu X and Her C: Physical and functional interaction between hMSH5 and c-Abl. Cancer Res 66: 151-158, 2006

62. Friedenson B: The BRCA1/2 pathway prevents hematologic cancers in addition to breast and ovarian cancers. BMC Cancer 7: $152,2007$.

63. Foray N, Marot D, Randrianarison V, Venezia ND, Picard D, Perricaudet M, Favaudon V and Jeggo P: Constitutive association of BRCA1 and c-Abl and its ATM-dependent disruption after irradiation. Mol Cell Biol 22: 4020-4032, 2002.

64. Schreiber V, Dantzer F, Ame JC and de Murcia G: Poly(ADP-ribose): Novel functions for an old molecule. Nat Rev Mol Cell Biol 7: 517-528, 2006.

65. Erdélyi K, Bakondi E, Gergely P, Szabó C and Virág L: Pathophysiologic role of oxidative stress-induced poly(ADP-ribose) polymerase-1 activation: Focus on cell death and transcriptional regulation. Cell Mol Life Sci 62: 751-759, 2005.

66. Malanga M and Althaus FR: The role of poly(ADP-ribose) in the DNA damage signaling network. Biochem Cell Biol 83: 354-364, 2005.

67. Haince JF, McDonald D, Rodrigue A, Déry U, Masson JY, Hendzel MJ and Poirier GG: PARP1-dependent kinetics of recruitment of MRE11 and NBS1 proteins to multiple DNA damage sites. J Biol Chem 283: 1197-1208, 2008.

68. Bonicalzi ME, Haince JF, Droit A and Poirier GG: Regulation of poly(ADP-ribose) metabolism by poly(ADP-ribose) glycohydrolase: Where and when? Cell Mol Life Sci 62: 739-750, 2005.

69. Bohio AA, Sattout A, Wang R, Wang K, Sah RK, Guo X, Zeng X, Ke Y, Boldogh I and Ba X: c-Abl-mediated tyrosine phosphorylation of PARP1 is crucial for expression of proinflammatory genes. J Immunol 203: 1521-1531, 2019. 